Classification

Physics Abstracts

$61.14 \mathrm{Dc}-61.16 \mathrm{Bg}$

\title{
Can the Multislice Method Be Used To Calculate HOLZ Reflections in High-Energy Electron Diffraction and Imaging?
}

\author{
Jiang Hua Chen, Marc Op de Beeck $\left(^{*}\right)$ and Dirk Van Dyck \\ EMAT, University of Antwerp (RUCA), Groenenborgerlaan 171, 2020 Antwerpen, Belgium
}

(Received October 2, 1995; accepted January 3, 1996)

\begin{abstract}
The validity of the use of the conventional multislice (MS) method for the calculation of higher-order Laue zone (HOLZ) reflections is tested by detailed calculations as well as a theoretical analysis. It is shown that if sufficiently thin slices are employed and the Debye-Waller (DW) factors for atoms are included the MS method can calculate HOLZ effects correctly up to the exact solution of the modified Schrödinger equation for high-energy electron diffraction. It is pointed out that the MS method is accurate up to second order in the slice thickness for zero-order Laue zone (ZOLZ) reflections but only up to first order for HOLZ reflections. As a consequence, the accurate calculation of HOLZ reflections requires a much smaller slice thickness. Using MS procedures in the standard way can then lead to accurate ZOLZ intensities but inaccurate HOLZ intensities. Without the introduction of DW factors for atoms, the atomic cores act as points of singularity for the MS formula and may cause severe errors for both the calculated HOLZ and ZOLZ reflections. It is also pointed out that if no so-called lower order HOLZ reflections are involved, one can simply use the projection approximation in HRTEM image simulations.
\end{abstract}

\section{Introduction}

The conventional multislice (MS) approach, which was originally proposed by Cowley and Moodie [1], has proven to be a good numerical procedure for solving the Schrödinger equation which describes the diffraction prccess [2-5]. However up till now, there is still a controversy whether the MS approach can properly include higher-order Laue zone (HOLZ) reflections [6-8]. It has been generally believed that if the crystal is cut into very thin slices, with the thickness $\varepsilon$ of each slice being a fraction of the periodicity $c$ in the incident beam direction, e.g., $\varepsilon<c / 2$, the HOLZ reflections would be described properly by the MS formula [6]. However, it was shown by Qin and Urban that although the variation of the crystal potential (for the case of $\mathrm{YBa}_{2} \mathrm{Cu}_{3} \mathrm{O}_{7-x}$ [001] zone axis diffraction) along the incident electron beam direction was taken into account by dividing each unit cell into six sub-slices, the MS calculation yielded results with severe errors, as

$\left({ }^{*}\right)$ Postdoc Researcher for the National Fund for Scientific Research (Belgium). 
compared with the experimental results and with the results of Bloch wave calculations [7]. Moreover, it has been shown that a special slicing scheme seems to be necessary for the MS method to include HOLZ reflections [9, 10] (although Ref. [10] was not attentively to discuss the special slicing scheme for including HOLZ reflections).

From previous investigations upon the MS formula for calculating HOLZ effects, a few problems still remain to be elucidated: (i) Theoretically, it is difficult to clearly show how the MS formula takes HOLZ reflections into account. This is important to use the MS method properly and to interpret the obtained results. (ii) Although HOLZ reflections can be generated by the calculations, the accuracy to which the HOLZ effects are included in the MS method still remains to be tested [6, 7]. (iii) The calculated zero-order Laue zone (ZOLZ) reflections seem to be independent on the slicing scheme but the calculated HOLZ reflections strongly depends on it [9]. (iv) It was argued by references [11-13] that the MS formula shows a poor ability to deal with heavy-atom materials, but a more quantitative criterion is still lacking. (v) It is well-known that the HOLZ reflections are extremely sensitive with the variation of temperature factors (Debye-Waller) [14], but this has never been discussed in detail.

In this work, following a theoretical analysis which shows how the MS method includes HOLZ effects, detailed calculations are made for the $\mathrm{YBa}_{2} \mathrm{Cu}_{3} \mathrm{O}_{7-x}$ [001] zone axis diffraction. It is shown that the MS formula can include the HOLZ effects correctly up to the exact solution of the modified Schrödinger equation for high-energy electron diffraction, if a sufficient number of slices is used and Debye-Waller factors are included for the atoms.

\section{Theory}

2.1 MS Method fOR Including HOLZ Reflections. - It is well-known that the MS method is developed for calculating high-energy electron diffraction which is based on the modified Schrödinger equation [15],

$$
\frac{\partial \Psi(\mathbf{r})}{\partial z}=\left[\frac{i \lambda}{4 \pi} \Delta+i \sigma V(\mathbf{r})\right] \Psi(\mathbf{r}),
$$

where $\lambda$ and $\sigma$, respectively, are the electron wavelength and the interaction constant, and $\Delta$ is the Laplacian operator in the $x-y$ plane.

To solve equation (1) for the transmission of the high-energy electron wave in a crystal, the crystal can be regarded as the combination of a series of crystal slices with equal thickness $\varepsilon$. The accurate exit wavefunction after the $n$th slice can therefore be written as [1]

$$
\Psi_{n}(\mathbf{R})=\hat{A}_{n} \hat{A}_{n-1} \ldots \hat{A}_{1} \Psi_{0}(\mathbf{R}),
$$

where $\hat{A}_{n}$ is the rigorous slice-transmission operator with

$$
\begin{aligned}
\hat{A}_{n}(\mathbf{R}) & =1+\int_{(n-1) \varepsilon}^{n \varepsilon}\left[\frac{i \lambda \Delta}{4 \pi}+i \sigma V(\mathbf{R}, z)\right] \mathrm{d} z+\ldots \\
& +\int_{(n-1) \varepsilon}^{n \varepsilon}\left[\frac{i \lambda \Delta}{4 \pi}+i \sigma V\left(\mathbf{R}, z_{1}\right)\right] \mathrm{d} z_{1} \int_{(n-1) \varepsilon}^{z_{1}} \ldots \int_{(n-1) \varepsilon}^{z_{k-1}}\left[\frac{i \lambda \Delta}{4 \pi}+i \sigma V\left(\mathbf{R}, z_{k}\right)\right] \mathrm{d} z_{k}+\ldots
\end{aligned}
$$

If within each slice, the potential variation along the $z$-direction is neglected, the slice-transmission operator $\hat{A}_{n}$ can be simplified as $\hat{B}_{n}[15,16]$ with:

$$
\hat{B}_{n}(\mathbf{R})=\mathrm{e}^{i \varepsilon\left[\frac{\lambda \Delta}{4 \pi}+\sigma V_{n}^{\mathrm{p}}(\mathbf{R})\right]}
$$


So we have

$$
\Psi_{n}(\mathbf{R})=\mathrm{e}^{i \varepsilon\left[\frac{\lambda \Delta}{4 \pi}+\sigma V_{n}^{\mathrm{p}}(\mathbf{R})\right]} \ldots \mathrm{e}^{i \varepsilon\left[\frac{\lambda \Delta}{4 \pi}+\sigma V_{1}^{\mathrm{p}}(\mathbf{R})\right]} \Psi_{0}(\mathbf{R}),
$$

where $\Psi_{0}(\mathbf{R})$ is the incident wavefunction (or boundary condition) which can be a plane wave or a coherent probe function [17] and $V_{n}^{\mathrm{p}}(\mathbf{R})$ is the average potential over slice $n$, defined by

$$
V_{n}^{\mathrm{p}}(\mathbf{R})=\frac{1}{\varepsilon} \int_{(n-1) \varepsilon}^{n \varepsilon} V(\mathbf{R}, z) \mathrm{d} z .
$$

Using the first-order Zassenhaus expansion [7] (which is accurate up to the first order in slice thickness), we obtain the MS method with the slice-transmission operator $\hat{M}_{n}$ :

$$
\begin{aligned}
\Psi_{n}(\mathbf{R}) & =\hat{M}_{n} \cdot \hat{M}_{n-1} \ldots \hat{M}_{1} \cdot \Psi_{0}(\mathbf{R}) \\
\hat{M}_{n} & =\mathrm{e}^{i \varepsilon \frac{\lambda \Delta}{4 \pi}} \cdot \mathrm{e}^{i \varepsilon \sigma V_{n}^{\mathrm{p}}(\mathbf{R})},
\end{aligned}
$$

A fact which is very important for following discussions is that the MS method can always be transformed into a second-order expansion of equation (5) by simply shifting the whole the crystal by half a slice [15]. This can easily be seen by rewriting the MS formula as

$$
\Psi_{n}(\mathbf{R})=\mathrm{e}^{\frac{1}{2}\left(i \varepsilon \frac{\lambda \Delta}{4 \pi}\right)} \hat{M}_{n}^{\prime} \cdot \hat{M}_{n-1}^{\prime} \ldots \hat{M}_{1} \cdot \mathrm{e}^{-\frac{1}{2}\left(i \varepsilon \frac{\lambda \Delta}{4 \pi}\right)} \Psi_{0}(\mathbf{R})
$$

with the second-order operator

$$
\hat{M}_{n}^{\prime}=\mathrm{e}^{\frac{1}{2}\left(i \varepsilon \frac{\lambda \Delta}{4 \pi}\right)} \cdot \mathrm{e}^{i \varepsilon \sigma V_{n}^{\mathrm{p}}(R)} \cdot \mathrm{e}^{\frac{1}{2}\left(i \varepsilon \frac{\lambda \Delta}{4 \pi}\right)} .
$$

For the calculation of diffraction beam intensities, the starting propagator and the ending propagator in equation (8) do not influence the final results, so that in that case the MS formula is effectively a second-order approximation of equation (5). It has also been shown by performing calculations in real space that equation (5) and the MS formula gave almost exactly the same results even for heavy materials as $\mathrm{Au}[001]$, with a slicing thickness of $2.037 \AA$ [18]. On the other hand, as we will see, the slicing thickness required for calculating HOLZ reflections with the MS method is much smaller than the usual slicing thickness. So we will base our analysis on a study of the solution of equation (5) in case of HOLZ interaction.

It should be addressed that operator $\hat{B}_{n}$ does not include any sources within the slice which introduce HOLZ effects, and from the mathematical point of view, it is only the first-order approximation of the exact operator $\hat{A}_{n}$. Up to the second-order approximation, we have

$$
\hat{A}_{n}(\mathbf{R})=\hat{B}_{n}(\mathbf{R})-\frac{\lambda \sigma \varepsilon^{2}}{8 \pi}\left[\left(\delta_{n} V_{n}\right) \Delta-\Delta\left(\delta_{n} V_{n}\right)\right]
$$

where $\delta_{n}(\mathbf{R})$ is the "potential eccentricity" which was introduced by Van Dyck to describe a second-order slice method [5]. The potential eccentricity takes values in the range from 0 to 1 .

In order to look for how equation (5) takes HOLZ effects into account, we can write in shorthand notation $\Delta=\frac{i \lambda}{4 \pi} \Delta$ and $V_{n}=i \sigma V_{n}^{\mathrm{p}}(\mathbf{R})$ and consider the transmission of the electron wave through two successive slices $i-1$ and $i$ :

$$
\begin{aligned}
\Psi_{i} & =\mathrm{e}^{\varepsilon\left(\Delta+V_{\imath}\right)} \cdot \mathrm{e}^{\varepsilon\left(\Delta+V_{\imath-1}\right)} \Psi_{i-2} \\
& =\left[\mathrm{e}^{\varepsilon\left(2 \Delta+V_{\imath}+V_{\imath-1}\right)}+\hat{H}_{\varepsilon}\left(V_{i}-V_{i-1}\right)\right] \cdot \Psi_{i-2}
\end{aligned}
$$


where $\mathrm{e}^{\varepsilon\left(2 \Delta+V_{2}+V_{1-1}\right)}$ only depends on the final projected potential of slices $i$ and $i-1$ and therefore refers to ZOLZ effect, so that the operator $\hat{H}_{\varepsilon}$ actually represents the generation of HOLZ effects due to the potential difference between slice $i$ and $i-1$, with

$$
\begin{aligned}
\hat{H}_{\varepsilon}\left(V_{i}-V_{i-1}\right) & =\frac{\varepsilon^{2}}{2}\left[\left(V_{i}-V_{i-1}\right) \Delta-\Delta\left(V_{i}-V_{i-1}\right)\right]+O\left(\varepsilon^{3}\right)+\ldots \\
& =-\frac{\varepsilon^{2}}{2}\left\{\left[\Delta\left(V_{i}-V_{i-1}\right)\right]+2\left[\nabla\left(V_{i}-V_{i-1}\right)\right] \cdot \nabla\right\}+O\left(\varepsilon^{3}\right)+\ldots
\end{aligned}
$$

where $\nabla$ is the gradient operator in the $x-y$ plane. We can clearly see from equation (11) and equation (12) that HOLZ effects are fundamentally generated by potential variation in the $z$-direction. If all the slices are the same, i.e. $V_{i}=\bar{V}(i=1,2, \ldots, n), \hat{H}_{\varepsilon}=0$ and no HOLZ effects are involved:

$$
\Psi_{n}(\mathbf{R})=\mathrm{e}^{n \varepsilon \Delta+\varepsilon \sum_{i=1}^{n} V_{i}} \Psi_{0}(\mathbf{R})=\mathrm{e}^{n \varepsilon(\Delta+\bar{V})} \Psi_{0}(\mathbf{R}) .
$$

Equation (13) is just the basis of the popular projection approximation which has been extensively used in HRTEM image simulations.

It should be noticed that the ZOLZ operator $\mathrm{e}^{\varepsilon\left(2 \Delta+V_{\imath}+V_{\imath-1}\right)}$ in equation (11) can continue to produce HOLZ effects when it is combined with other ZOLZ operators from subsequent slices. In order to see this clearly, we now use equations (11) and (12) to obtain the MS transmission operator $\hat{T}_{\mathrm{c}}$ of one unit cell which is assumed to be divided into $2^{n}$ slices. $\hat{T}_{\mathrm{c}}$ is eventually found to be

$$
\begin{aligned}
\hat{T}_{c}= & \mathrm{e}^{c(\Delta+\bar{V})}+\hat{H}_{c / 2}\left(V_{22}-V_{21}\right)+\mathrm{e}^{\frac{c}{2}\left(\Delta+V_{22}\right)} \hat{H}_{c / 4}\left(V_{42}-V_{41}\right) \\
& +\hat{H}_{c / 4}\left(V_{44}-V_{43}\right) \mathrm{e}^{\frac{c}{2}\left(\Delta+V_{21}\right)}+\hat{H}_{c / 4}\left(V_{44}-V_{43}\right) \hat{H}_{c / 4}\left(V_{42}-V_{41}\right) \\
& +f_{8}\left(\hat{H}_{c / 8}\right)+\ldots+f_{2^{n}}\left(\hat{H}_{c / 2^{n}}\right),
\end{aligned}
$$

where $c$ is the crystal periodicity along the $z$-direction, $V_{m i}$ indicates the average potential over the $i$-th slice in case the unit cell is divided into $m$ slices, so that we can use relations such as

$$
\sum_{i=1}^{m} V_{m i}=m \bar{V}, \quad V_{m 2}+V_{m 1}=2 V_{\frac{m}{2} 1}, \ldots,
$$

where $\bar{V}=\frac{1}{c} \int_{0}^{c} V(R, z) \mathrm{d} z$. In equation $(14), f_{8}\left(\hat{H}_{\mathrm{c} / 8}\right)$ represents the HOLZ effects contributed by pairs of successive slices and their "propagation" in case the unit cell is divided into 8 slices (Fig. 1), with

$f_{8}\left(\hat{H}_{c / 8}\right)=\mathrm{e}^{\frac{c}{2}\left(\Delta+V_{22}\right)} \mathrm{e}^{\frac{c}{4}\left(\Delta+V_{42}\right)} \hat{H}_{c / 8}\left(V_{82}-V_{81}\right)+\hat{H}_{c / 4}\left(V_{44}-V_{43}\right) \mathrm{e}^{\frac{c}{4}\left(\Delta+V_{42}\right)} \hat{H}_{c / 8}\left(V_{82}-V_{81}\right)+\ldots$.

Similarly, with more complicated expressions, $f_{2^{n}}\left(\hat{H}_{c / 2^{n}}\right)$ indicates the HOLZ effects contributed by pairs of successive slices and their "propagation" in case the unit cell is divided into $2^{n}$ slices. From the point of view of the multislice approach, Figure 1 shows the constitution of HOLZ effects contributed by one unit cell.

Equation (14) also shows how the MS method works in the inclusion of HOLZ reflections:

(1) Calculated HOLZ effects are strongly depend on the slicing schemes: the MS procedure would yield different HOLZ results by using different slice thicknesses until sufficiently thin slices are employed. 


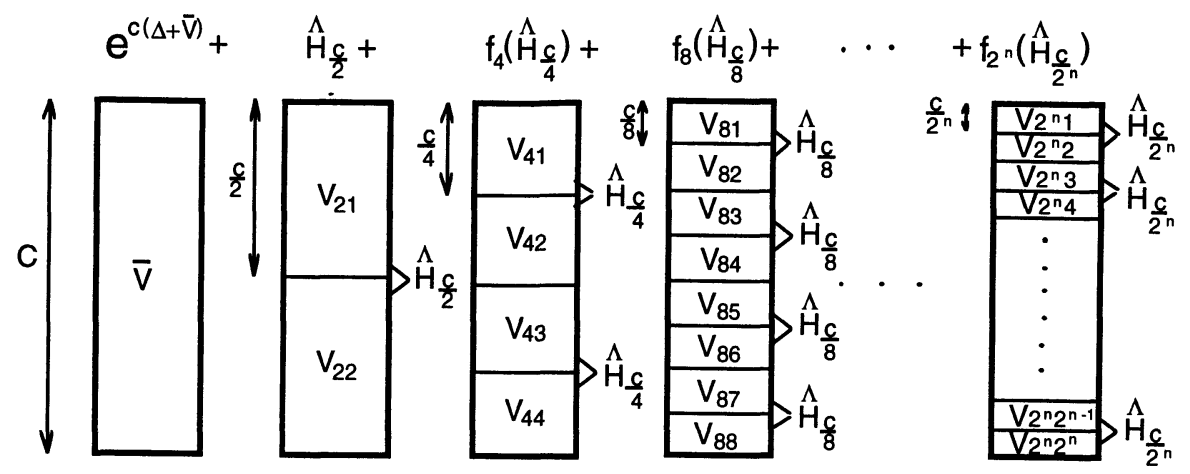

Fig. 1. - Illustration of the constitution of the HOLZ effects contributed by one unit cell-from the point of view of the multislice method.

(2) Although it neglects the HOLZ effects within each of the slices, the MS method includes the HOLZ effects due to potential variations between slices.

(3) The larger the slicing number is, the more accurate the calculated HOLZ lines are. To see whether the slicing thickness is small enough, in principle we can estimate the last term of equation (14) as follows

$$
\left|f_{2^{n}}\left(\hat{H}_{c / 2^{n}}\right) \Psi_{0}\right| \ll 1
$$

(4) The ZOLZ operator of one unit cell, $\mathrm{e}^{c(\Delta+\bar{V})}$, depends only on the final projected potential of the unit cell and will not be able to continue to produce HOLZ effects when combined with other operators of subsequent unit cells, since $\mathrm{e}^{c(\Delta+\bar{V})} \cdot \mathrm{e}^{c(\Delta+\bar{V})}=\mathrm{e}^{2 c(\Delta+\bar{V})}+\hat{H}_{c}(\bar{V}-$ $\bar{V})=\mathrm{e}^{2 c(\Delta+\bar{V})}$.

To see the final HOLZ effects, we set $\hat{T}_{c}=\mathrm{e}^{c(\Delta+\bar{V})}+\hat{h}_{c}$, where $\hat{h}_{c}$ indicates the HOLZ eff $\epsilon$ cts from one unit cell. As compared with the ZOLZ operator, $\hat{h}_{c}$ is small $\left(\propto \lambda^{2}\right)$, so that we can suppose that in the following equation, terms as $\hat{h}_{c} \cdot \hat{h}_{c}\left(\propto \lambda^{4}\right)$, which represent multiple HOLZ scattering, can be neglected. Approximately, the final wavefunction after $N$ unit cells can be expressed as

$$
\begin{aligned}
\Psi_{n}(\mathbf{R}) & =\left(\mathrm{e}^{c(\Delta+\bar{V})}+\hat{h}_{c}\right)^{N} \Psi_{0}(\mathbf{R}) \\
& \approx \mathrm{e}^{N c(\Delta+\bar{V})}+\sum_{k=1}^{N} \mathrm{e}^{(N-k) c(\Delta+\bar{V})} \cdot \hat{h}_{c} \cdot \mathrm{e}^{k c(\Delta+\bar{V})} \Psi_{0}(\mathbf{R})
\end{aligned}
$$

Equation (18) shows that the final HOLZ effects depends both on the contributions of each unit cell as well as on interferences between the cells-after "propagation" through the crystal. This means that the HOLZ effects always exist within each unit cell but those effects from different unit cells may cancel each other and finally give no observable HOLZ effects. In case no HOLZ reflections appear, one can simply use the popular projection approximation. 
the incident wavefunction (boundary condition) by recursive operations of the following equation:

$$
\Psi_{n}(R, z=n \varepsilon)=\mathrm{e}^{\frac{i \lambda \varepsilon \Delta}{4 \pi}} \cdot \mathrm{e}^{i \sigma \varepsilon V_{n}^{\mathrm{p}}(R)} \Psi_{n-1}[R, z=(n-1) \varepsilon]
$$

where $\mathrm{e}^{\frac{i \lambda \varepsilon \Delta}{4 \pi}}$ is the so-called Fresnel propagator [17]. In reciprocal space, the Fresnel propagator appears in the following form

$$
P(h, k)=\mathrm{e}^{-\pi i \lambda \varepsilon\left(\frac{h^{2}}{a^{2}}+\frac{k^{2}}{b^{2}}\right)} .
$$

In equation (19) $\mathrm{e}^{\imath \sigma \varepsilon V_{n}^{\mathrm{p}}(\mathbf{R})}$ is the phase grating in which $\mathbf{R}=(x, y)$.

In equation (19), every unit cell of the crystal is assumed to be evenly divided into a few thin slices perpendicular to the $z$-direction. If the crystal with lattice parameters $a, b$ and $c$ is orthogonal and the each unit cell contains $m$ slices, one has $\varepsilon=c / m, V_{n}^{\mathrm{p}}(\mathbf{R})$ can be calculated from the atomic scattering amplitudes of each atom through the following relationship [19]:

$$
V_{n}^{\mathrm{p}}(\mathbf{R})=\frac{C}{\Omega_{\text {cell }}} \sum_{h, k} U_{n}(h, k) \cdot \mathrm{e}^{2 \pi i\left(\frac{h x}{a}+\frac{k y}{b}\right)}
$$

where $C$ is a constant, $\Omega_{\text {cell }}$ is the volume of the unit cell and $U_{n}(h, k)$ is calculated by

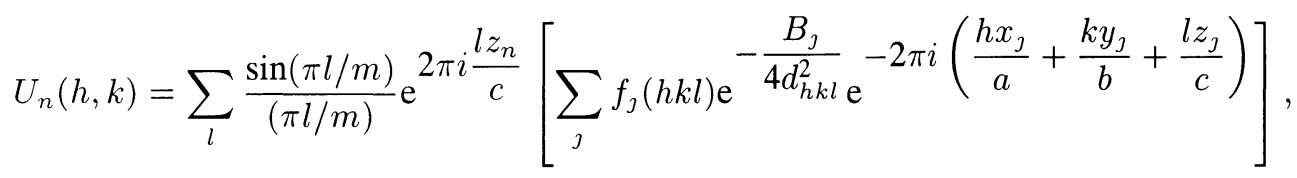

where $z_{n}=(n-1) \varepsilon+\varepsilon / 2, h, k$ and $l$ are Miller indices for the reflection $(h k l) .\left(x_{j}, y_{j}, z_{j}\right)$ and $B_{\jmath}$, respectively, are the co-ordinates and the Debye-Waller factor (DW) of atom $j$. In addition, $d_{h k l}$ is the interplanar spacing of reflection planes $(h k l)$. The atomic scattering factors in equation (22) are calculated by (see, e.g. Ref. [20])

$$
f_{\jmath}(h k l)=\sum_{\imath=1}^{4} \frac{a_{i \jmath}\left(1-\mathrm{e}^{-b_{\imath \jmath} / 4 d_{h k l}^{2}}\right)}{\left(1 / 4 d_{k_{\imath l} l}^{2}\right)}
$$

where the coefficients $a_{i j}$ and $b_{i j}$ for different atoms can be found in reference [21].

For ZOLZ calculations one only includes $l=0$ whereas $l \neq 0$ accounts for the HOLZ.

In the present calculations for $\mathrm{YBa}_{2} \mathrm{Cu}_{3} \mathrm{O}_{7-x}$ [001], equation (22) is used only for a single unit cell but the obtained phase gratings are used repeatedly for all the unit cells. It should be noted that this can be done if no lower order HOLZ reflections, e.g. the bulk-forbidden but surfaceallowed reflections, are involved [8, 9]. In other words, when lower order HOLZ reflections are involved, the top-bottom effects should be taken into account by using different phase gratings for both the top and the bottom slices. In the appendix of this paper, it is shown that the top-bottom effects are not important in the case of $\mathrm{YBa}_{2} \mathrm{Cu}_{3} \mathrm{O}_{7-x}[001]$ zone axis diffraction. 
Table I. - Structural data and Debye-Waller factors for $\mathrm{YBa}_{2} \mathrm{Cu}_{3} \mathrm{O}_{6.91}$ crystal which are used for calculations. The first group of $D W$ factors are taken from reference [22].

\begin{tabular}{|c|c|c|c|c|c|c|c|c|c|}
\hline \multicolumn{2}{|c|}{ type of atoms } & Y & $\mathrm{Ba}$ & $\mathrm{Cu} 1$ & $\mathrm{Cu} 2$ & $\mathrm{O} 1$ & $\mathrm{O} 2$ & $\mathrm{O} 3$ & $\mathrm{O} 4$ \\
\hline \multicolumn{2}{|c|}{ number of atoms } & 1 & 2 & 1 & 2 & 2 & 2 & 2 & 1 \\
\hline \multicolumn{2}{|c|}{ atom positions: } & $\frac{1}{2}, \frac{1}{2}, \frac{1}{2}$ & $\frac{1}{2}, \frac{1}{2}, z_{1}$ & $0,0,0$ & & & $\frac{1}{2}, 0, z_{4}$ & $0, \frac{1}{2}, z_{4}$ & $0, \frac{1}{2}, 0$ \\
\hline \multirow{4}{*}{\multicolumn{2}{|c|}{$\begin{array}{c}(x, y, z) \\
a=0.38177 \mathrm{~nm} \\
b=0.38836 \mathrm{~nm} \\
c=1.16827 \mathrm{~nm}\end{array}$}} & & $\frac{1}{2}, \frac{1}{2}, 1-z_{1}$ & & $0,0,1-z_{2}$ & $0,0,1-z_{3}$ & $\frac{1}{2}, 0,1-z_{4}$ & $0, \frac{1}{2}, 1-z_{4}$ & \\
\hline & & & & & & & & & \\
\hline & & & & & & & & & \\
\hline & & & $z_{1}=0.1844$ & & $z_{2}=0.3552$ & $z_{3}=0.1588$ & $z_{4}=0.3779$ & & \\
\hline \multirow{3}{*}{$\begin{array}{l}\text { DW } \\
\text { factors }\end{array}$} & 1 & 0.49 & 0.64 & 0.51 & 0.52 & 0.75 & 0.62 & 0.64 & 1.12 \\
\hline & 2 & 0.25 & 0.32 & 0.26 & 0.26 & 0.38 & 0.31 & 0.32 & 0.56 \\
\hline & 3 & 0.0 & 0.0 & 0.0 & 0.0 & 0.0 & 0.0 & 0.0 & 0.0 \\
\hline
\end{tabular}

\section{Calculations}

A FFTMS program written by the authors is used and calculations are carried out for the $\mathrm{YBa}_{2} \mathrm{Cu}_{3} \mathrm{O}_{7-x}[001]$ zone axis diffraction.

First of all, we check how the diffraction intensities change with decreasing the slice thickness. A series of slice thicknesses are used: $c / 4, c / 6, c / 8, c / 16, c / 32, c / 64(c=11.6827 \AA)$. Secondly, we check how the DW factors of atoms influence the calculated diffraction intensities. Three groups of the DW factors listed in Table I are used, The first group with the DW factors at room temperature are given by reference [22] form X-ray experiments. For the second group, we take 50\% of the tabulated values of the first group, so as to approximate the DW factors at lower temperature. The last group with the zero value for all the atoms corresponds to the MS calculation without including the DW factors. In addition, we also check the relations between the calculated HOLZ effects and the projected potential in the core area of heavy atoms such as $\mathrm{Ba}$ with respect to different slicing schemes.

The structural data of $\mathrm{YBa}_{2} \mathrm{Cu}_{3} \mathrm{O}_{7-x}$ from reference [22] are listed in Table I. The accelerating voltage of $200 \mathrm{kV}$ and the number of $64 \times 64$ sampling points (corresponding to the sampling intervals of $0.060 \AA \times 0.061 \AA$ ) in the $x-y$ plane are kept fixed for all calculations. The sum for $l$ in equation (22) is made over the range from $l=-40$ to $l=39$.

\section{Results}

The calculated results are given in Figure 2 through Figure 15. Figure 2 to Figure 4 and Figure 9 show the amplitudes of diffraction (at the crystal thickness of 40 c-units, about $467.32 \AA$ ) in the form of diffraction patterns, where a zero amplitude value is set for all the reflections whose intensities are less than $9.0 \times 10^{-6}$ and a fixed maximum amplitude value of $7.0 \times 10^{-2}$ is set for those which have intensities larger than $4.9 \times 10^{-3}$. Figure 5 to Figure 8 and Figure 10 to Figure 15, however, show amplitudes of reflections with their absolute values plotted against the crystal thickness. 
Fig. 2. - Calculated diffraction patterns from $\mathrm{YBa}_{2} \mathrm{Cu}_{3} \mathrm{O}_{7-x}$ [001], which show the changes of HOLZ reflection patterns from one slicing case to another. The letters "MSDWR" marked in the figure represent "multislice method with including the Debye-Waller (DW) factors at room temperature" and " $n$ " is the slicing number in one unit cell.

Figure 2 through Figure 8 show that the calculated intensities (amplitudes) of HOLZ reflections change with decreasing slice thickness, dramatically from $c / 4$ down to $c / 16$, slightly from $c / 16$ to $c / 32$, but remain nearly constant from $c / 32$ to $c / 64$. This means that sufficiently thin slices are required in order to include the HOLZ effects properly in the MS method. In the present case, a thickness of less than $c / 32(0.365 \AA)$ should be used.

It is shown by Figure 9 through Figure 11 that large differences appear between the results with and without DW factors. Only calculations including DW factors are in agreement with experimental data, i.e. the HOLZ reflections are very weak [6]. Moreover, not only the HOLZ reflections but also the ZOLZ reflections are strongly influenced by the DW factors. It is obviously shown that HOLZ reflections could be stronger by cooling the sample.

Figure 12 shows that ZOLZ reflections without including DW factors change slightly with decreasing the slice thickness, but those including DW factors are almost exactly independent on the slicing scheme.

Figure 13 and Figure 14 show the projected potential (multiplied by the interaction constant) of the slice which contains the heavy atoms $\mathrm{Ba}$. Without including the DW factors, the potential distribution in the core area of atom $\mathrm{Ba}$ is very sharp and the peak value is very high-even in the case of using very thin slices, e.g. with a thickness of $c / 64(0.183 \AA)$. The projected potential, however, becomes smooth and lower when DW factors are included. 
MS[MiT: $n=04$

MSDinT: $r=16$ mint: $n=06$

Mscint: $r=08$

Fig. 3. - The same as for Figure 2 but with including the DW factors at lower temperature.

MS: $n=04$

mis $r_{1}=16$

$M 5 r=3$ ms $n=08$

$M S n=64$

Fig. 4. - The same as for Figure 2 but without including the DW factors. 


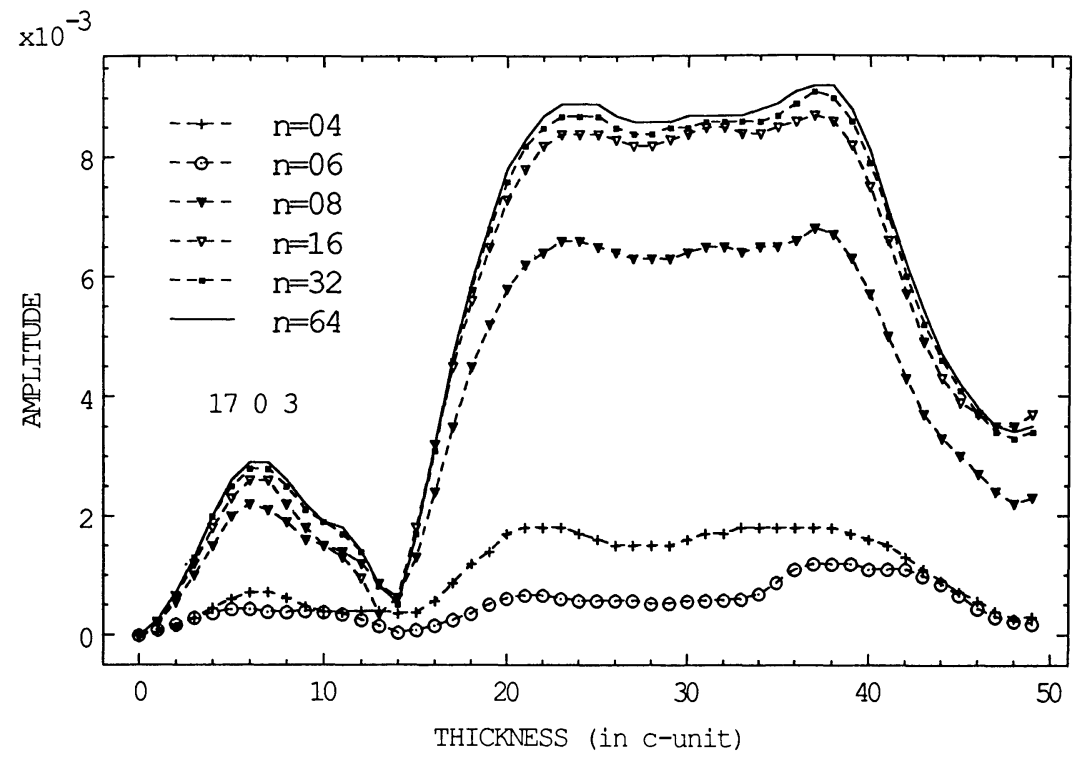

Fig. 5. - Amplitudes of (17 03 3) reflection against the crystal thickness calculated with including the DW factors at room temperature, and definitions of the lines used to depict the curves obtained from different slicing cases.

\section{Discussions}

The appearance of HOLZ reflections depends on the scattering power of the atoms, the periodicity in the beam direction, the incident direction as well as the wavelength of electron wave. HOLZ effects exist within each of the unit cells, but contributions of all the unit cells may well cancel each other so as not to generate strong resonant HOLZ reflections. However, if particular illumination conditions are chosen, crystals with small $c$ parameters can also give strong resonant HOLZ reflections, as in the case of CBED.

The so-called lower order HOLZ reflections [9] mentioned before in fact are not common resonant HOLZ reflections but the bulk-forbidden, surface-allowed reflections from the top and the bottom atomic layers [8], which appear at the forbidden positions of the ZOLZ plane [8, 10]. This type of HOLZ reflections may cause observable effects in the HRTEM images, as shown in reference $[8,10]$. When this type of HOLZ reflections are involved, the crystal should be regarded as a non-periodic object in the $z$-direction. The top and bottom atomic layers can be different to those inside of the crystal and the effects should be included by using separate top-bottom phase gratings in the MS HRTEM image simulation. Although reference [8] presents a rigorous treatment for calculating phase gratings of 3D objects, in the appendix of this paper, a faster procedure is proposed in model II for treating such kind of calculations since inside the crystal the potential still repeats itself after each unit cell. So for the HRTEM image simulations of crystals, if lower order HOLZ reflections are involved, the rigorous MS procedure has to be done. If it is not the case, however, one can simply use the projection approximation since the large-angle resonant HOLZ sports with weak intensities do not directly contribute to the image.

Theoretically, we have seen that the accuracy, to which HOLZ reflections are included in the MS calculation, depends on how fine the slicing is made. For accurate calculations, the slicing thickness should be so small that the calculated results do not change any more by using thinner 

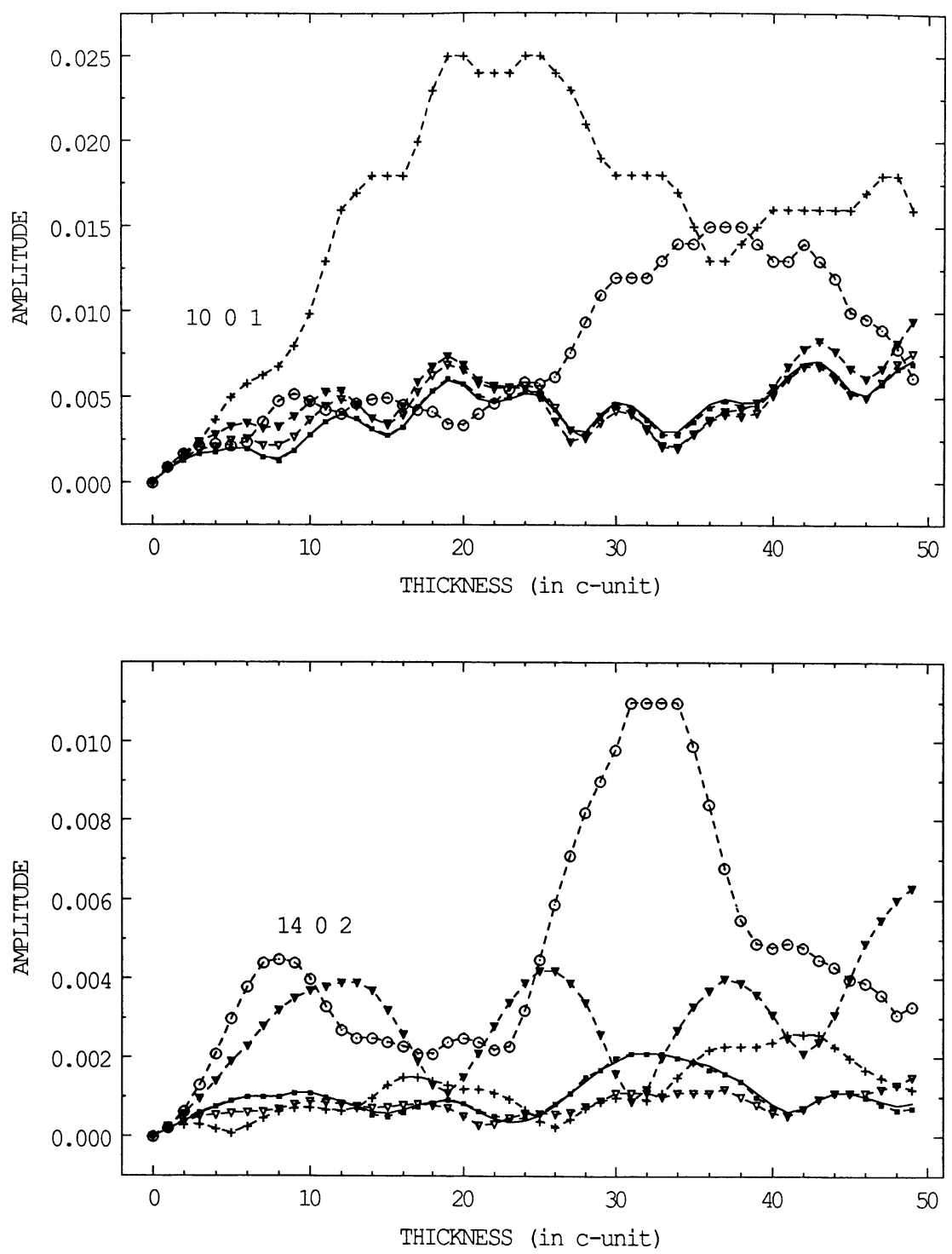

Fig. 6. - Amplitudes of (10 01 1) and (14 $\left.0 \begin{array}{ll}1 & 0\end{array}\right)$ reflections against the crystal thickness calculated with including the DW factors at room temperature, and definitions of the curves are given in Figure 5.

slices. It is shown by obtained results that the slicing-thickness requirements for including HOLZ reflections are much more severe than that for the usual cases where no HOLZ reflections are involved-usually a slicing thickness of no less than $1 \AA$ is sufficient for most of such cases [19]. This is understandable since the MS formula includes ZOLZ effects correctly up to the second order. So the MS formula with large slicing thicknesses could give, at the same time, correct intensities of ZOLZ reflections but wrong intensities of HOLZ reflections. This is just the case shown in Figure 12, where ZOLZ reflections are found being independent on the slicing thickness. But this is correct only if the total intensity of HOLZ reflections is not too strong and the overlap region between ZOLZ and HOLZ reflections is not too large. 

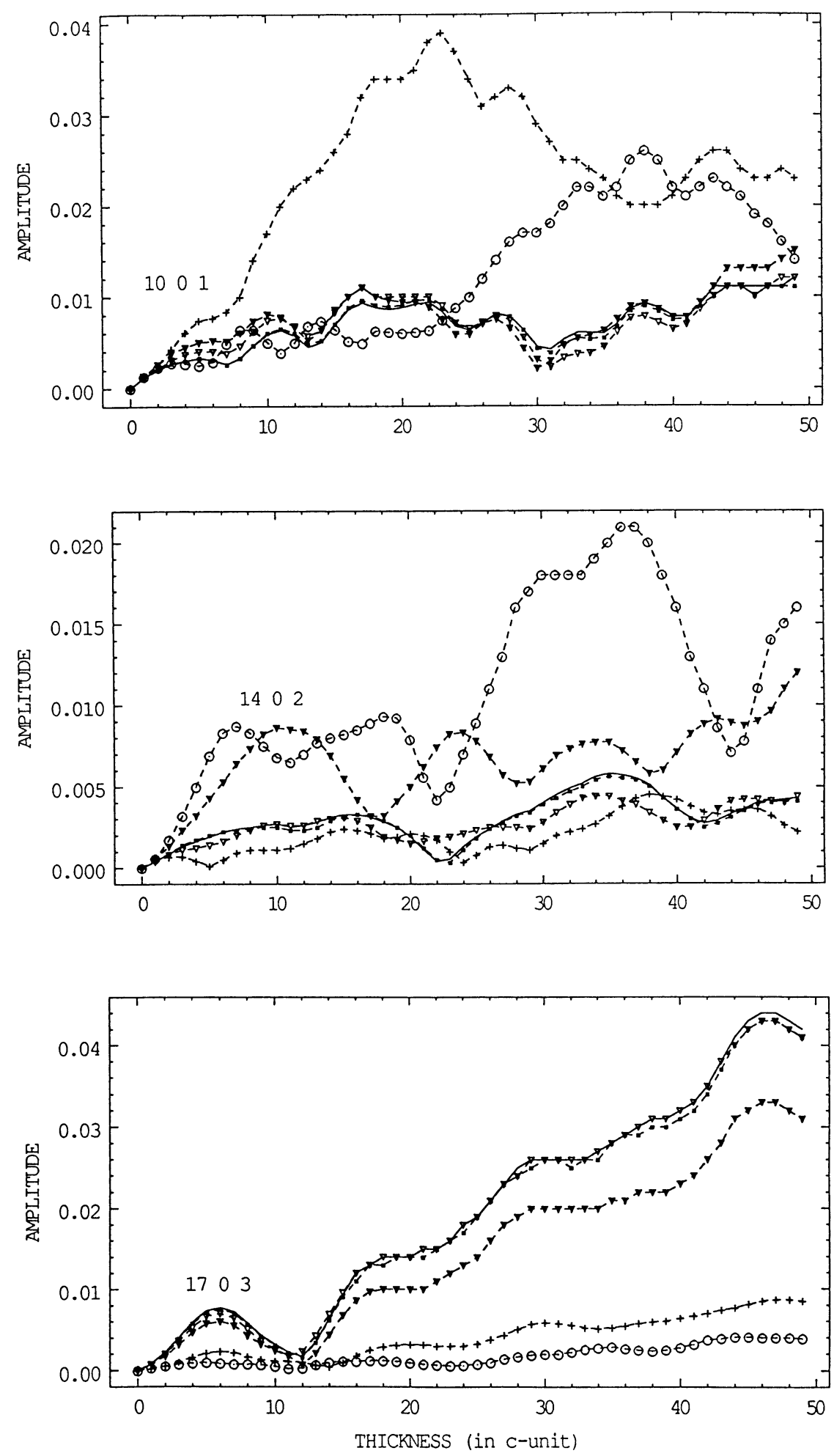

Fig. 7. - Amplitudes of (10 01 1), (14 02 2) and (17 03 ) reflections against the crystal thickness calculated with including the DW factors at lower temperature, and definitions of the curves are given in Figure 5. 

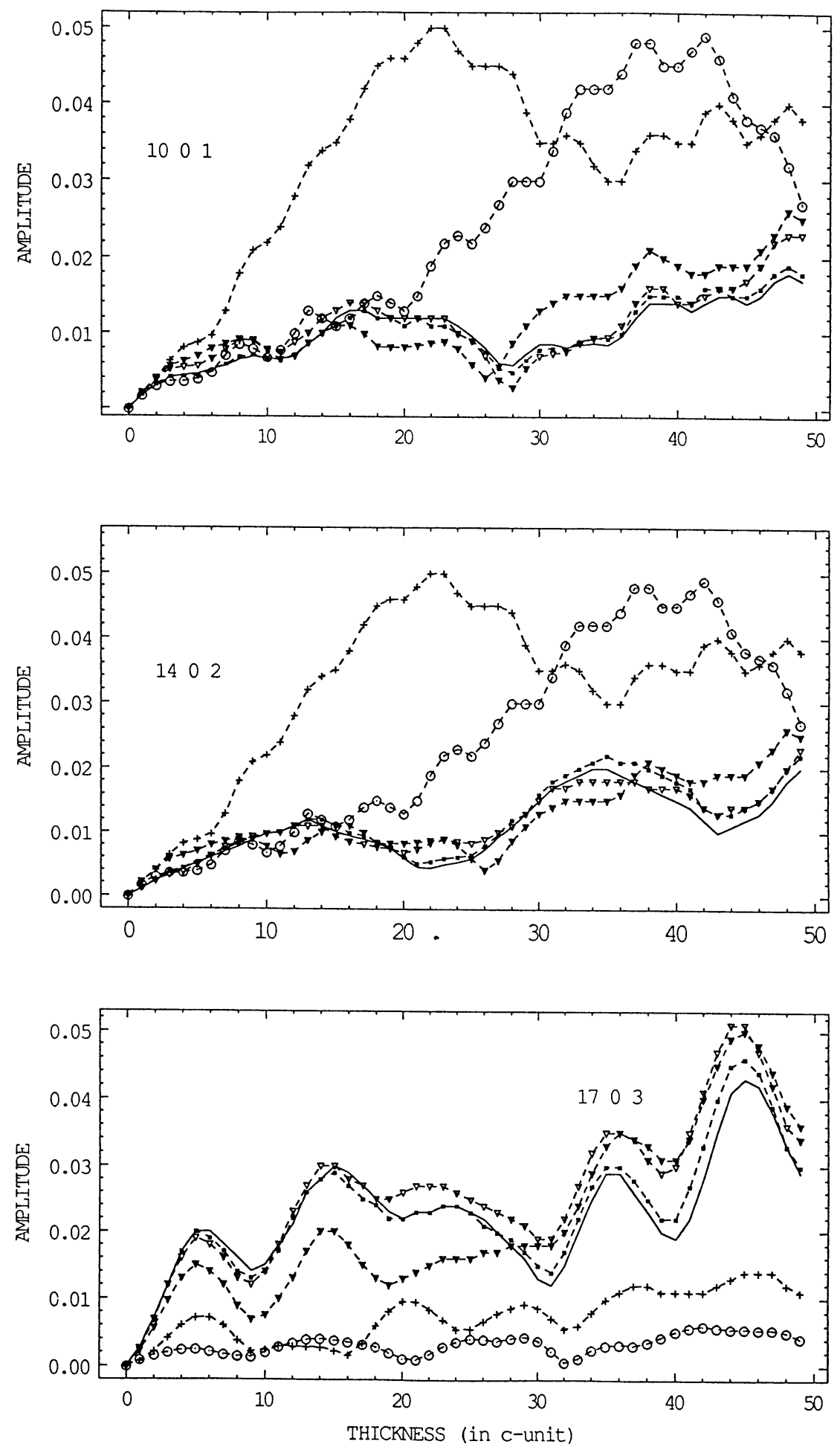

Fig. 8. - The same as for Figure 7 but without including the DW factors. 
Fig. 9. - Comparisons between diffraction patterns calculated with and without including the DW factors, where $n=01(/ 16)$ indicates taking $c$ as the slicing thickness for calculating projected potential and then cutting the projected potential in one unit cell into 16 slices for the recursive MS operations, in other words, $n=01(/ 16)$ is the case HOLZ reflections are neglected. $n=64$ indicates that the slicing number in one unit cell is 64. The letters "MSDWR", "MSDWT" and "MS", respectively, represent " multislice method with the DW factors at room temperature, lower temperature and without the DW factors.

Up to the second-order approximation, the relation between the MS operator and the exact slice-transmission operator is expressed in equation (10). We can clearly see that if the potential eccentricities of all the slices are zero, the MS formula automatically becomes of second-order for the inclusion of HOLZ effects. This is why references [4] and [5] suggest a special slicing scheme in which atomic centers are always at the centers of related slices. But this slicing scheme is not suitable for complex structures. In order to obtain a criterion which is able to clearly show the relationship between the crystal structure and the slicing thickness with which the MS formula can correctly calculate HOLZ reflections, we can in principle start from equation (17). But more theoretical work has to be done due to the complexity of the HOLZ operator $f_{2^{n}}\left(\hat{H}_{c / 2^{n}}\right)$.

Without including the DW factors, the results calculated by the MS formula are totally wrong (Fig.4). The projected potential in the core area of heavy atom $\mathrm{Ba}$, as we see in Figures 13 and 14 , is too sharp to decrease by decreasing slice thickness. The positions at which atomic nuclei, especially heavy atomic nuclei, are located, are the points of singularity for the MS method: projected potentials at these points can not be decreased to desirable values by using very small but finite slice thicknesses. This therefore results in severe errors in the obtained results. Fortunately, atomic nuclei are always in motion due to thermal vibration-even at zero temperature, and there- 

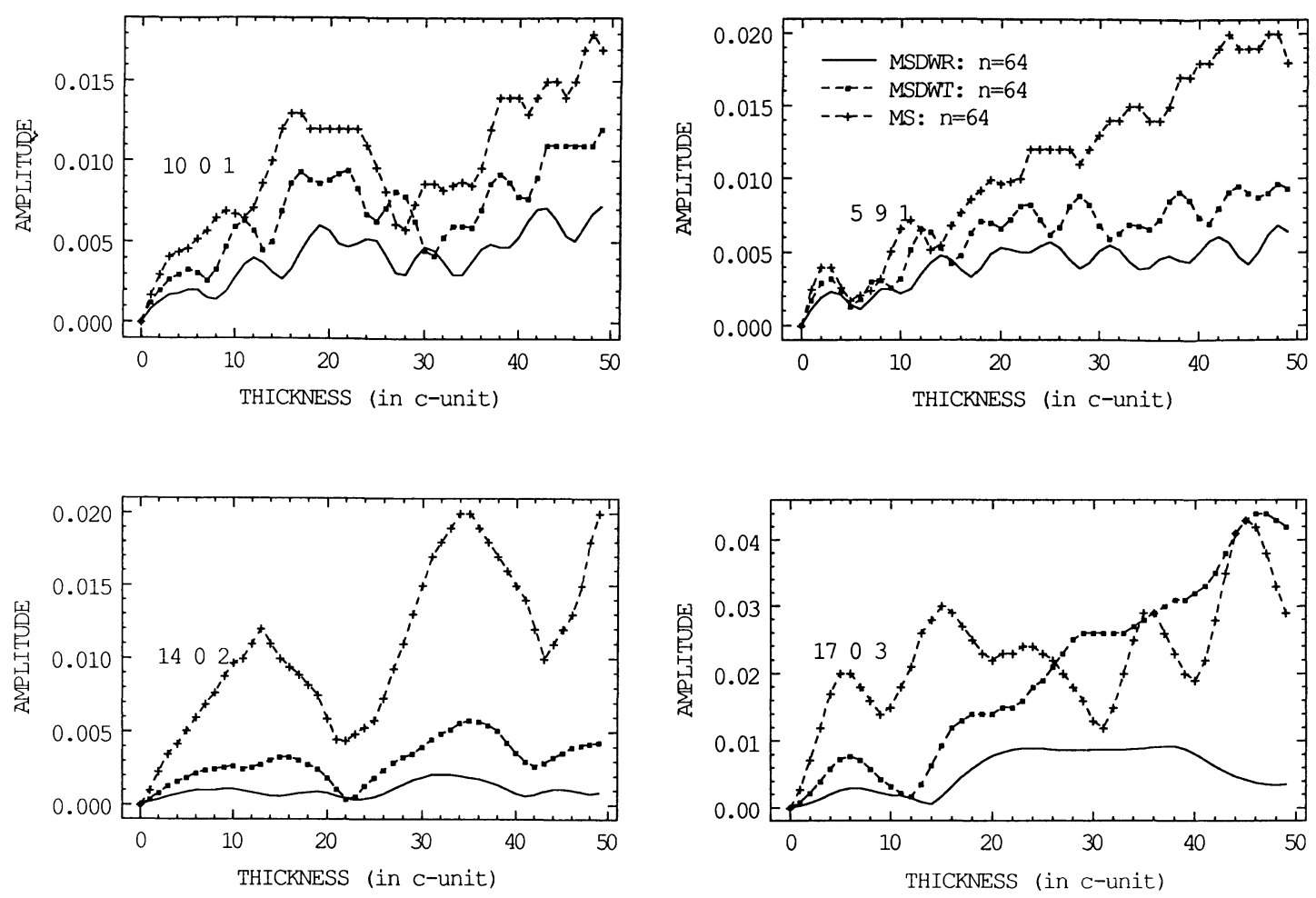

Fig. 10. - Amplitudes of HOLZ reflections against thickness, showing the differences between results calculated with and without including DW factors. The meanings of symbols is the same as in Figure 9.

fore the potential distribution in the core area of atoms should be convoluted by the probability function of thermal vibration: $\left(\frac{2 \sqrt{\pi}}{B_{J}}\right)^{3} \mathrm{e}^{-\frac{4 \pi^{2} r^{2}}{B_{j}}}$, where $B_{j}$ is the DW factor. This makes it possible for the MS formula to overcome the computational divergency at its points of singularity. In other words, with including the DW factors, the potential distribution becomes smooth and projected potentials in core areas of atoms can be decreased by using small slice thickness.

HOLZ effects, as it is shown by equation (12), are directly related to charge densities and potential gradients, and therefore mainly appear at large-angle reflection positions. The sharper the potential distribution in the crystal is, the stronger the HOLZ reflections are. Hence cooling sample usually leads to the appearance of more HOLZ reflections [14]. It should be noticed that ZOLZ reflections are also obviously influenced by temperature factors: the extinction distance becomes shorter when cooling the sample (Fig. 12). However, it must be noted that the temperature can approach zero but the DW factors can never be zero due to the zero point vibration.

To calculate HOLZ reflections accurately with the MS method, one has to include the DW factors of atoms and use sufficiently thin slices. This, however, immediately gives rise to two problems for practical MS calculations. One is how to get the DW factors for unknown structures (of course, this is a common problem for not only the MS but also other procedures) [23]. Another problem is that the severe slicing-thickness requirement for including HOLZ reflections make it much more time-consuming to perform MS calculations. To find a faster procedure for the calculation of HOLZ reflections, the second-order slice method proposed by Van Dyck will be investigated in detail in our next paper. 

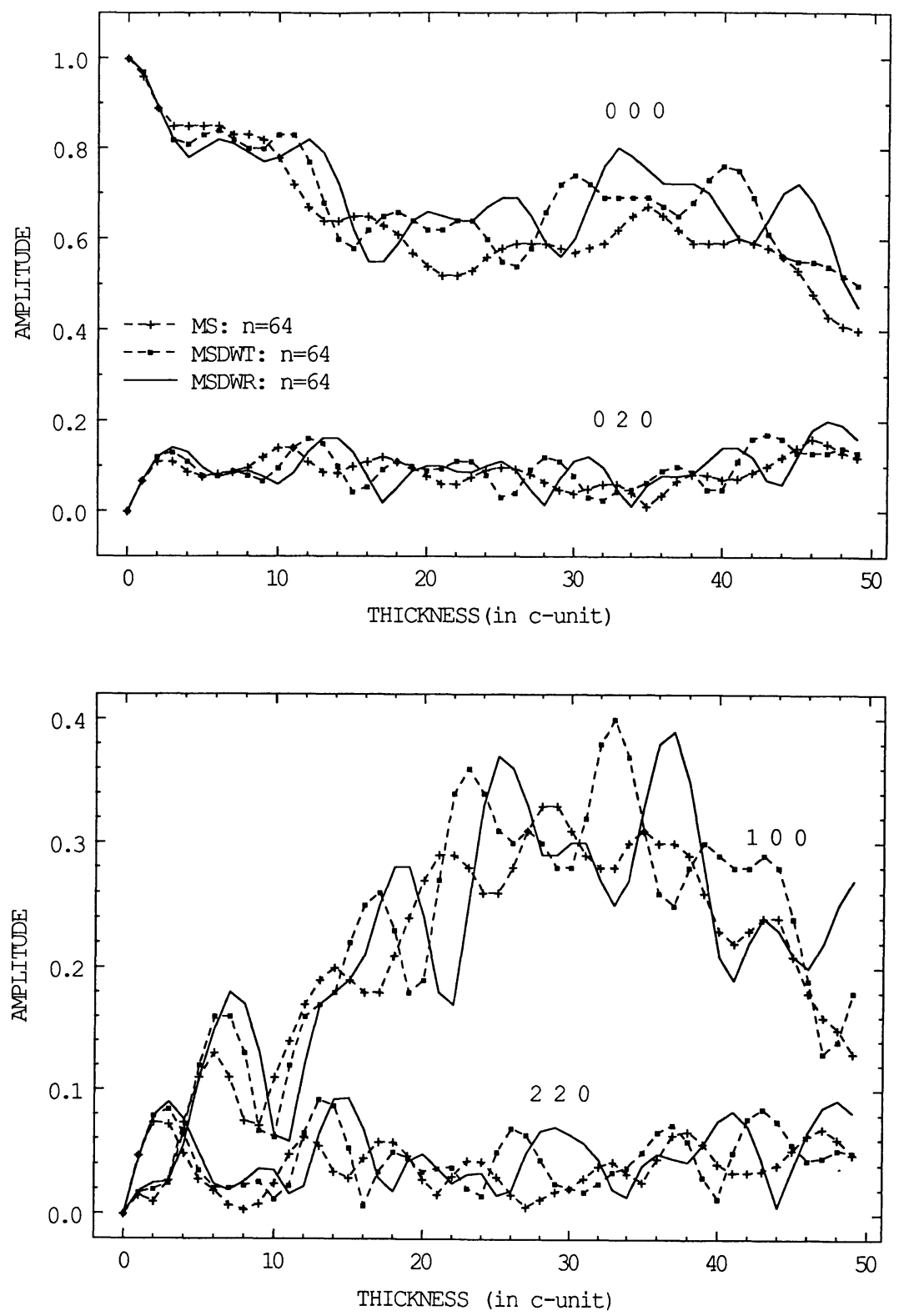

Fig. 11. - Amplitudes of ZOLZ reflections against thickness, showing the differences between results calculated with and without including DW factors. The meanings of symbols is the same as in Figure 9.

Finally, it should be noticed that by using parabola instead of sphere dispersion surfaces [24], equation (1) could cause errors for including large-angle resonant HOLZ effects. This effects perhaps could be corrected by simple modifications of the excitation error in the Fresnel propagator given in equation (20) (see the appendix of Ref. [6] and the definition of the propagator in Ref. [19] ). However, such corrections still need to be tested or proved. 

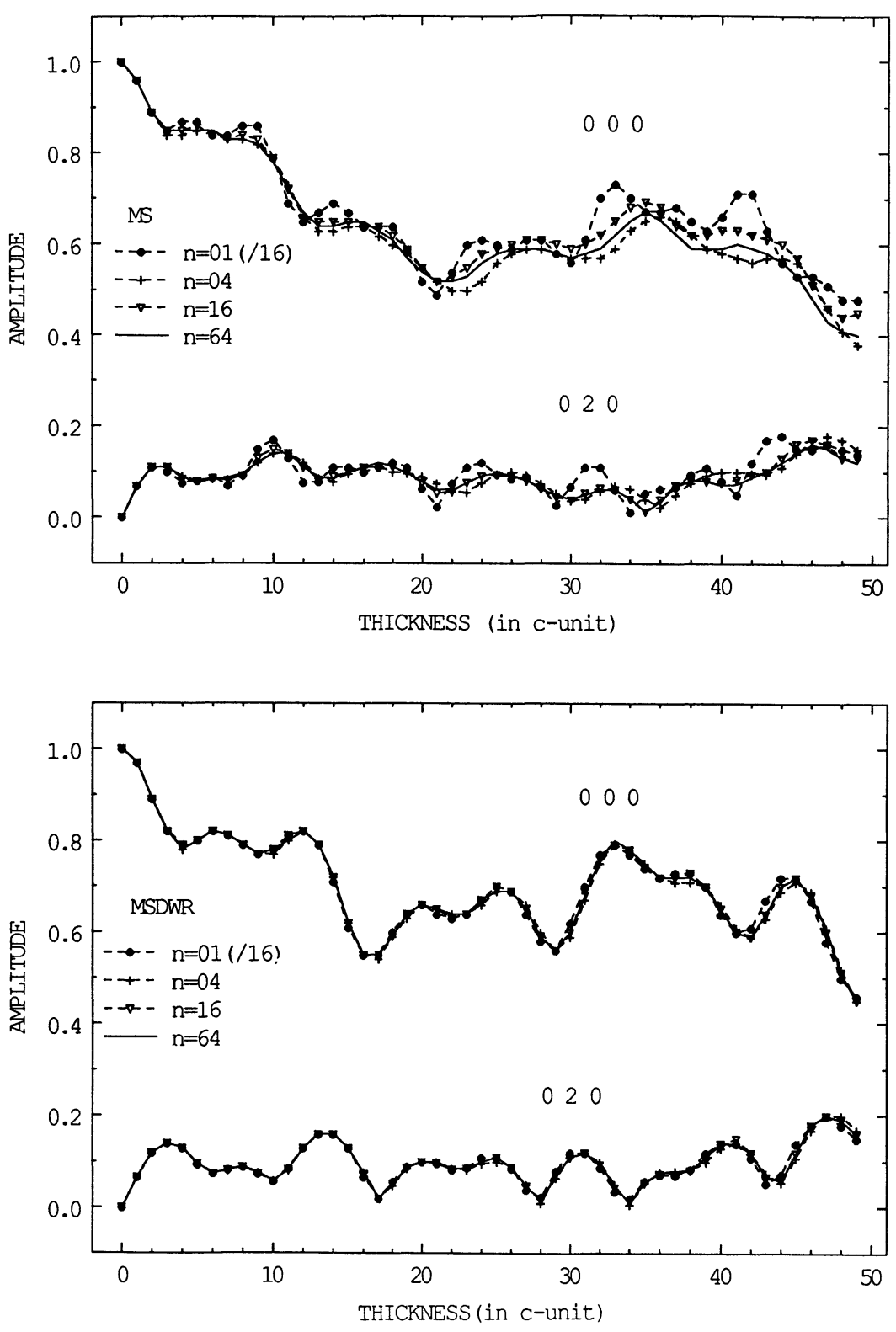

Fig. 12. - Amplitudes of ZOLZ reflections against thickness, showing that ZOLZ reflections are almost independent on slicing schemes. Results in the top figure (marked by MS) are calculated without including the DW factors.

\section{Conclusions}

Sufficiently fine slicing in the $z$-direction for including HOLZ effects is as important as the number of sampling points in the $x-y$ plane for calculating ZOLZ reflections. The DW factors should always be included in the MS procedure for accurate calculations not only of HOLZ but also ZOLZ reflections. 

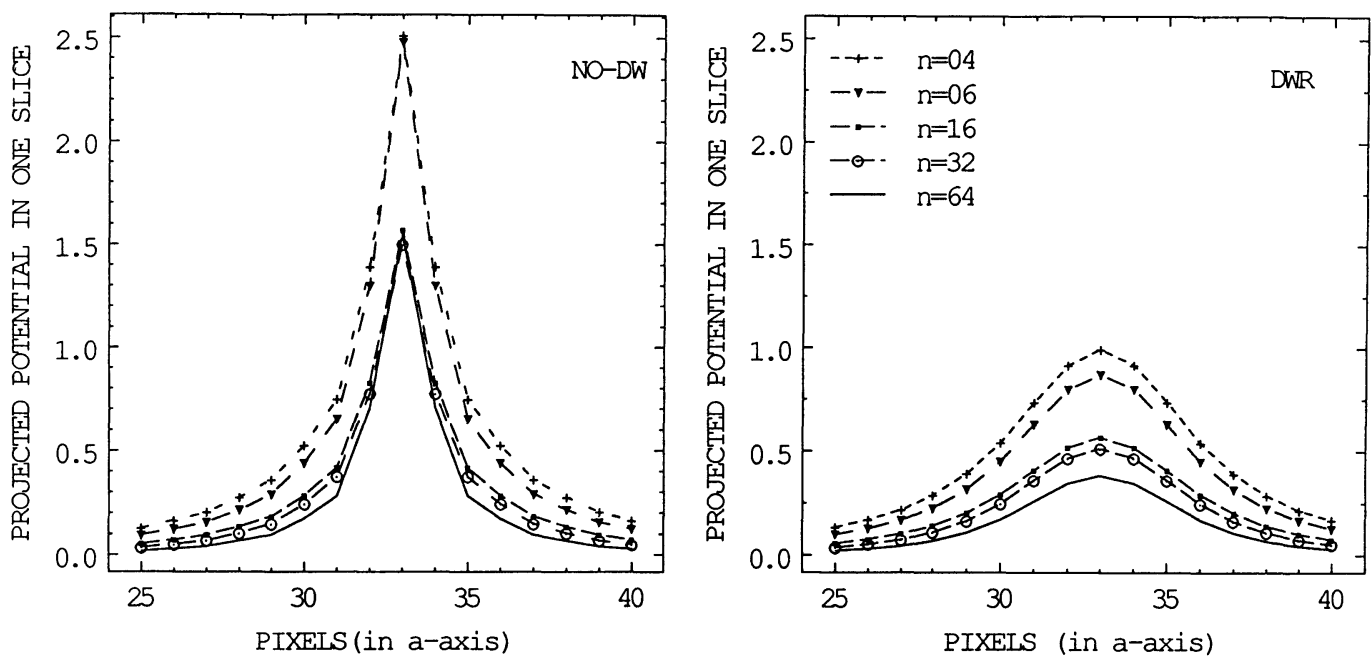

Fig. 13. - Distributions of the projected potential of the slice which contains the center of atom Ba, showing the smoothing of potential by including the DW factors.

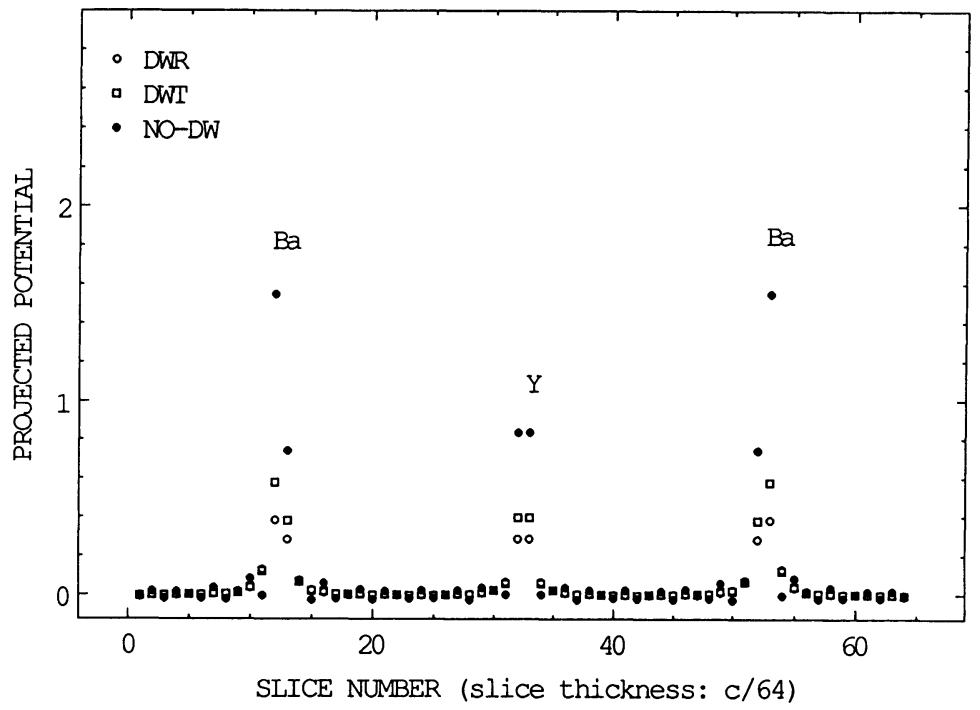

Fig. 14. - Distribution of the projected potential values of all the slices along the $z$-axis which passes exactly through the center of $\mathrm{Ba}-\mathrm{Y}-\mathrm{Ba}$ atomic column.

The MS formula is only a first-order approximation of the rigorous solution of the basic equation, but it automatically becomes a second-order method when no HOLZ reflections are involved. Hence, the slicing thickness for the MS formula to include HOLZ reflections is much smaller than that to calculate ZOLZ reflections.

Without the introduction of DW factors, atomic centers act as singularities for the MS formula and may lead to severe errors, e.g. producing HOLZ reflections which are much stronger than experimental ones. With including the DW factors, however, this difficulty can be overcome. 


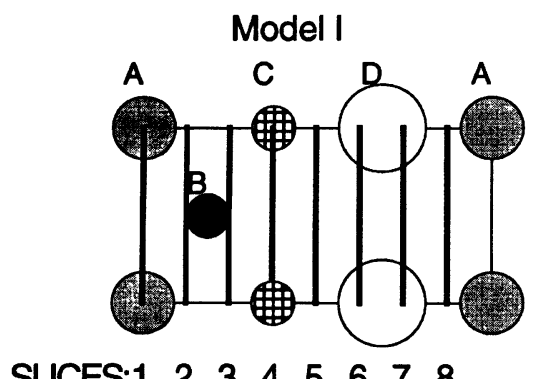

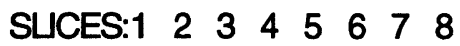

Model II

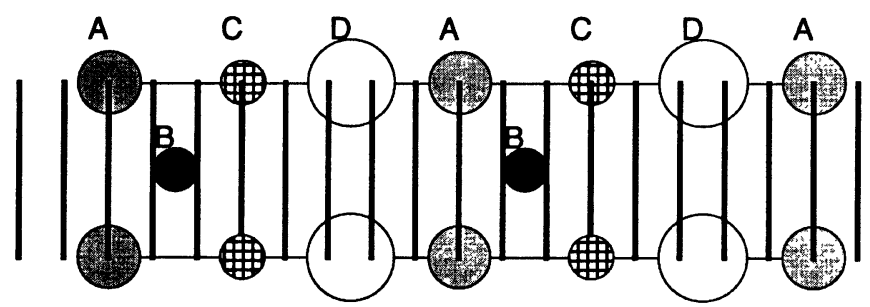

SUCES:1 $23 \quad 3 \quad 4 \quad 5 \quad 6 \quad 78991011121314151617181920$

a)
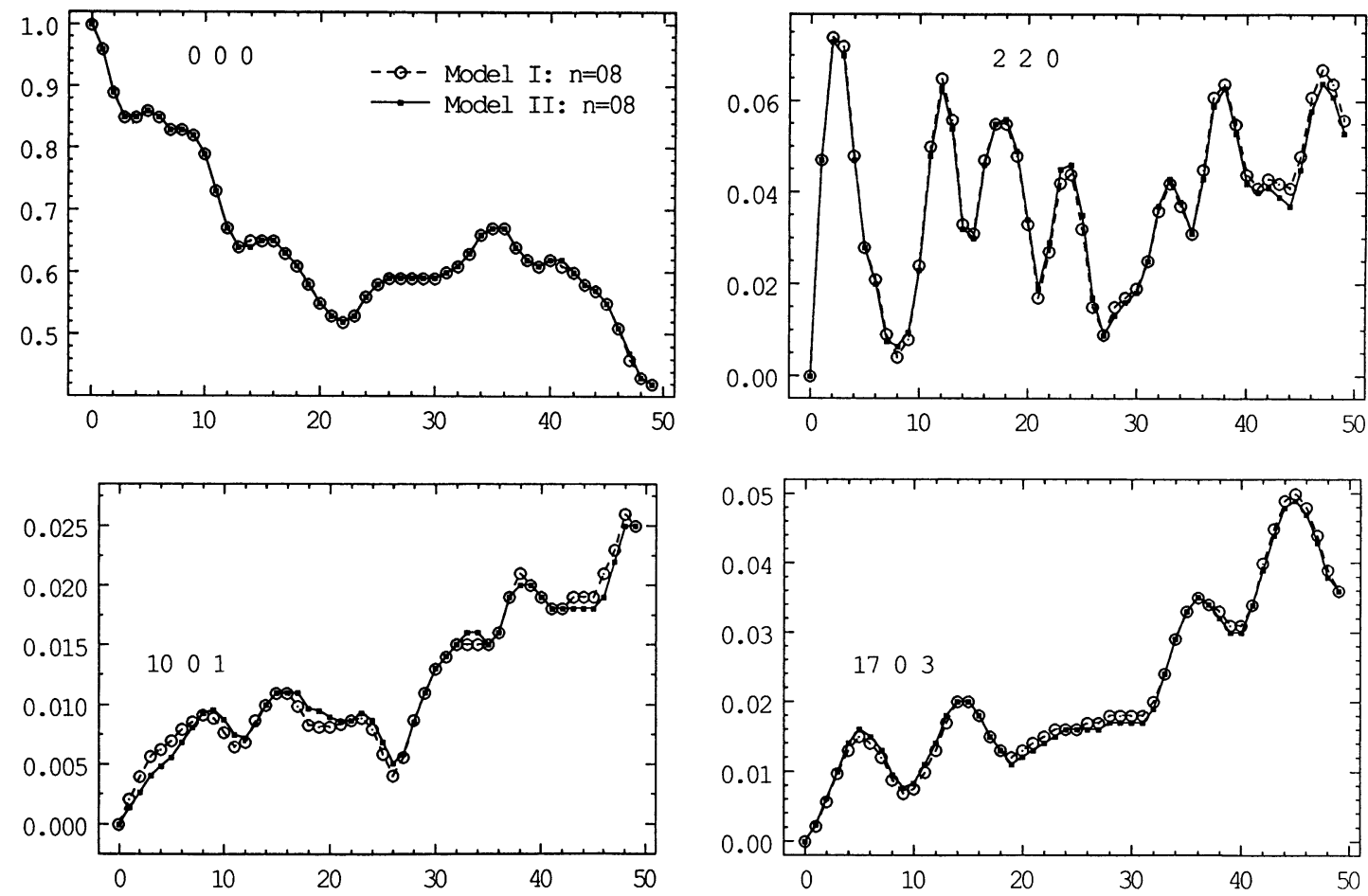

b)

Fig. 15. - a) Illustration of two slicing models. b) Comparisons between results calculated with two different slicing models. 
For the HRTEM imaging of crystals, there are two types of HOLZ effects. One is the lower order HOLZ reflections which may directly contribute to the images. Another is the large-angle resonant HOLZ reflections which do not cause observable effects in the images. In case there is no lower order HOLZ sports, one can simply use the projection approximation for the HRTEM image simulations.

\section{Acknowledgements}

This text presents research results partly sponsored by the Belgian programme on InterUniversity Poles of Attraction initiated by the Belgian State, Prime Minister's Office of Science Policy Programming. The scientific responsibility is assumed by the authors. The authors wish to thank Prof. J. Van Landuyt for his enduring support.

\section{Appendix}

There are two models in Figure 15a to describe a crystal for MS calculations. Model I is the one which is popular and is used in the present work. However, model II is more rigorous to a real crystal because the top and bottom slices are calculated separately. When performing MS calculations with model II, we start with top slices-1, 2, 3, 4, 5, 6, but repeat with bulk slices-7, $8,9,10,11,12,13,14$, and then end the calculation with bottom slices-15, 16, 17, 18, 19, 20. The results calculated with model I and model II are compared in Figure 15b. As we can see from the obtained results, the top-bottom effects are not important in our case.

\section{References}

[1] Cowley J.M. and Moodie A.F., Acta Cryst. 10 (1957) 609.

[2] Goodman P. and Moodie A.F., Acta Cryst. A30 (1974) 280.

[3] Ishizuka K. and Uyeda N., Acta Cryst. A33 (1977) 740.

[4] Van Dyck D., Phys. Status Solidi 72 (1975) 321.

[5] Van Dyck D., J. Microsc. 119 (1980) 141.

[6] Kilaas R., O’Keefe M.A. and Krishnan K.M., Ultramicrosc. 21 (1987) 47.

[7] Qin L.C. and Urban K., Ultramicrosc. 33 (1990) 159.

[8] Marks L.D., Ultramicrosc. 38 (1992) 325.

[9] Self P.G. and O'Keefe M.A., in High-Resolution Transmission Electron Microscopy and Associated Techniques (Oxford University Press, 1992) p. 259.

[10] Nishio K., Isshiki T., Saijo H. and Shiojiri M., Ultramicrosc. 54 (1994) 301.

[11] Lynch D.F., Acta Cryst. A27 (1971) 399.

[12] Watanabe K., Kikuchi Y., Hiratsuka K. and Yamaguchi H., Phys. Status Solidi (a) 109 (1988) 119.

[13] Watanabe K., Mitsuishi K. and Hashimoto I., Phys. Status Solidi (b) 181 (1994) 23.

[14] Steeds J.W., in Introduction to Analytical Electron Microscopy, chapter 15 (Plenum Press, New York and London, 1979).

[15] Van Dyck D., Adv. Elec. Elec. Phys. (1985) 295.

[16] Coene W. and Van Dyck D., Ultramicrosc. 15 (1984) 41. 
[17] Chen J.H., Van Dyck D., Op De Beeck M., Broeckx J. and Van Landuyt J., Phys. Status Solidi (a) 150 (1995) 13.

[18] Chen J.H., (1993) unpublished work.

[19] Self P.G., O’Keefe M.A., Buseck P.R. and Spargo A.E.C., Ultramicrosc. 11 (1983) 35.

[20] Spence J.C.H. and Zuo J.M., Electron Microdiffraction (Plenum Press, New York and London, 1992) p. 309.

[21] Inter. Tables For X-Ray Cryst., Vol. IV (The Kynoch Press, 1974) p. 99.

[22] Yvon K. and Francois M., Z. Phys. B-Condensed Matter 75 (1989) 1-32.

[23] Inter. Tables For X-Ray Cryst., Vol. III (The Kynoch Press, 1964) p. 232.

[24] Bird D.M., J. Elec. Mic. Tech. 13 (1989) 77. 